Research Paper

\title{
Areca Nut Chewing and Risk of Atrial Fibrillation in Taiwanese Men: A Nationwide Ecological Study
}

\author{
Wei-Chung Tsai ${ }^{1,2}$, Chung-Yu Chen ${ }^{3}$, Hsuan-Fu Kuo', Ming-Tsang Wu 4,5, Wei-Hua Tang 1, Chih-Sheng \\ Chu 1, Tsung-Hsien Lin 1, Ho-Ming Su 1,2, Po-Chao Hsu ${ }^{1}$, Shih-Jie Jhuo ${ }^{1}$, Ming-Yen Lin ${ }^{5}$, Kun-Tai Lee ${ }^{1}$, \\ Sheng-Hsiung Sheu ${ }^{1}$, Wen-Ter Lai ${ }^{1}$ \\ 1. Division of Cardiology, Department of Internal Medicine, Kaohsiung Medical University Hospital, Kaohsiung Medical University, \\ Kaohsiung, Taiwan; \\ 2. Department of Internal Medicine, Kaohsiung Municipal Hsiao-Kang Hospital, Kaohsiung Medical University, Kaohsiung, Taiwan; \\ 3. College of Pharmacy, Kaohsiung Medical University, Kaohsiung, Taiwan; \\ 4. Department of Family Medicine, Kaohsiung Medical University Hospital, Kaohsiung, Taiwan; \\ 5. Department of Public Health, Kaohsiung Medical University, Kaohsiung, Taiwan.
}

$\square$ Corresponding author: Kun-Tai Lee, Department of Internal Medicine, Kaohsiung Medical University, Kaohsiung, No.100, Shih-Chuan 1st Rd., Sanmin District, Kaohsiung City 807, Taiwan (R.O.C.). Tel: (886)(7) 3121101 EXT. 2315 Fax: (886)(7) 3234845 E-mail: 920265@mail.kmuh.org.tw.

(c) Ivyspring International Publisher. This is an open-access article distributed under the terms of the Creative Commons License (http://creativecommons.org/ licenses/by-nc-nd/3.0/). Reproduction is permitted for personal, noncommercial use, provided that the article is in whole, unmodified, and properly cited.

Received: 2013.0I.3I; Accepted: 2013.04.15; Published: 2013.04.25

\begin{abstract}
Background: Areca nut chewing is associated with the risk of obesity, metabolic syndrome, hypertension, and cardiovascular mortality. Although a few case reports or case series have suggested the link between areca nut chewing and cardiac arrhythmias, information about the relationship between areca nut chewing and atrial fibrillation (AF) is lacking. Thus, a nationwide ecological study was conducted to investigate this.

Methods: Two national datasets, the nationwide population-based 2005 Taiwan National Health Insurance Research dataset (NHIRD) and the 2005 National Health Interview Survey (NHIS), were used for analyses. The clinical characteristics, inhabited area and medical histories for 375,360 eligible males were retrieved from the 2005 NHIRD. Health related behaviors including areca nut chewing, cigarette smoking, infrequent vegetable eating, and exercise habit were collected from the $2005 \mathrm{NHIS}$. The prevalence of AF and the areca nut chewing rate were evaluated by multivariate analysis.

Results: Of the 375,360 males (mean age, 44 years old), I,326 (0.35\%) were diagnosed with AF. The higher areca nut chewing rate, the higher prevalence rate of AF in Taiwan (Spearman correlation coefficient $r=0.558, p=0.007$ ). After adjusting for other covariates, the current areca nut chewing rate was found to be independently associated with the prevalence of AF. The adjusted odd ratio for areca nut chewing was $1.02(95 \% \mathrm{Cl}=1.00-1.04)$ in risk of $\mathrm{AF}$ prevalence.

Conclusions: Areca nut chewing is independently associated with the prevalence of AF in Taiwanese men. However, further exploration of the underlying mechanisms is necessary.
\end{abstract}

Key words: atrial fibrillation, areca nut chewing.

\section{Introduction}

Atrial fibrillation/atrial flutter (AF) is the most commonly diagnosed atrial tachyarrhythmia in clinical practice [1]. According to The Framingham Heart Study, subjects with AF had an increased risk of stroke and a near two-fold mortality rate as compared to those without [2]. In Taiwan, the overall prevalence rate of $\mathrm{AF}$ was $1.07 \%$ [3]. Taiwanese patients with $\mathrm{AF}$ had a four-fold and two-fold increased risk of suffer- 
ing from stroke and all-cause-death, respectively [3].

Areca nut (AN) chewing is the fourth most popular substance abuse habit in the world [4]. Previous studies have found that AN chewing is associated with the risk of obesity, metabolic syndrome, hypertension, cardiovascular disease such as coronary artery disease (CAD), and all-cause mortality [5-10]. Several case reports/case series also show that AN chewing may be linked to cardiac arrhythmias [11-16]. However, to our knowledge, there is no study investigating the relationship between $\mathrm{AN}$ chewing and AF. Thus, we conducted a nationwide ecological study by combining the population-based National Health Insurance research dataset (NHIRD)[17] and the National Health Interview Survey (NHIS)[18] to examine the association between $\mathrm{AF}$ and AN chewing. Due to the lower AN chewing rate in Taiwanese women, male subjects only were analyzed [19].

\section{Methods}

\section{Study population}

Study subjects were randomly selected from 2004-2005 NHIRD; all were males and older than 18 years of age. Subjects residing in off-shore islands were excluded due to differences in life style and health related behaviors (Figure 1). Taiwan's National Health Insurance (NHI) program enrolled about 23 million people, $\sim 99 \%$ of the Taiwanese population. The NHIRD contained data on utilization of all NHI resources, including outpatient visits, hospital care, and prescribed medications. The study cohort was identified and tracked from a subset of the NHIRD, the Longitudinal Health Insurance Database of 2005 [18]. Demographic and medical data including age, gender, residential area, and co-morbidity were obtained from the 2004-2005 NHIRD. The co-morbidities were obtained by diagnosis according to the International Classification of Diseases, Ninth Revision (ICD9) codes of outpatient visits or hospitalizations.. The co-morbidities predisposing subjects to AF were selected and used in multivariate analysis (Supplemental table 1). Since the NHIRD consists of encrypted secondary data, the study was waived from full review by the Institutional Review Board in Kaohsiung Medical University Hospital.

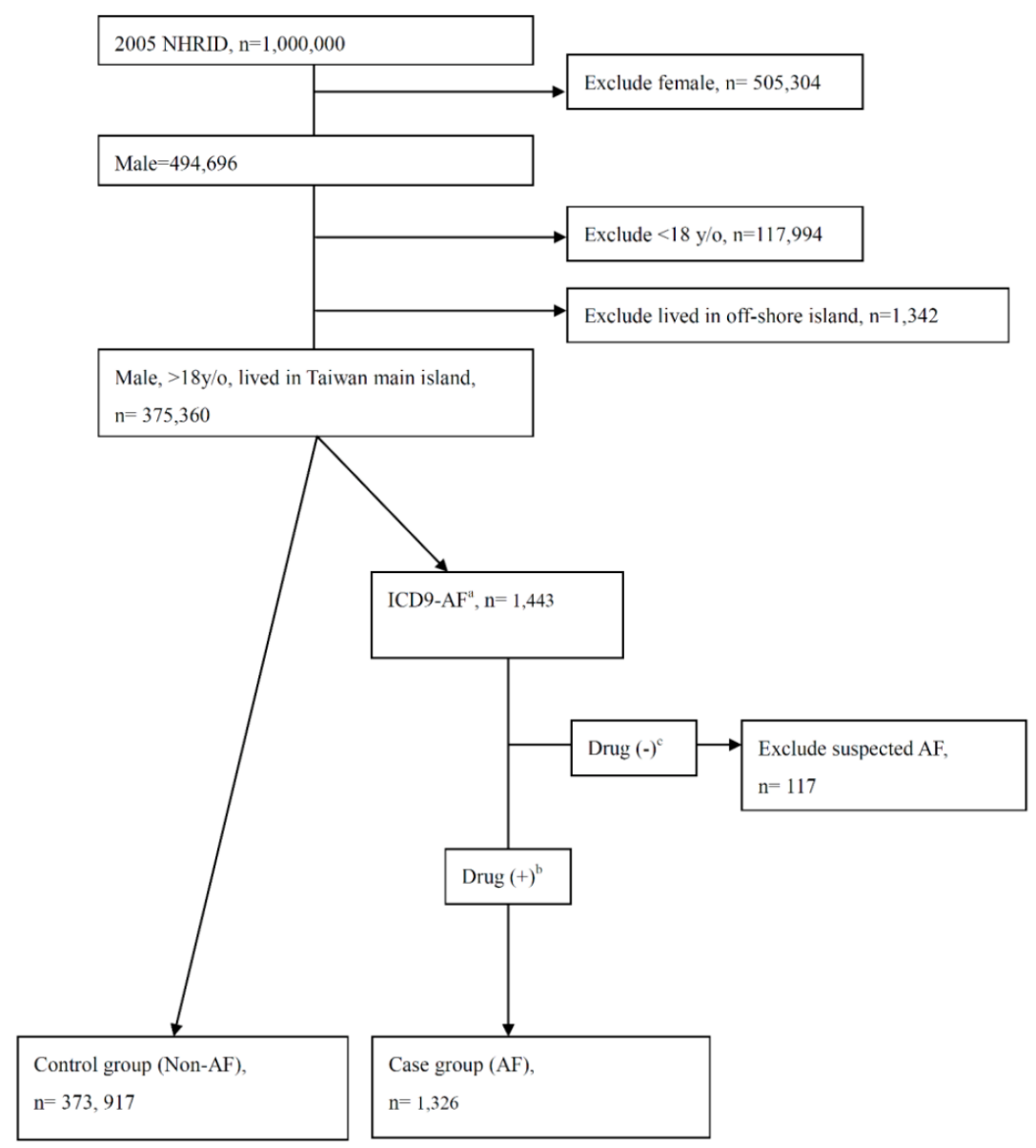

Figure I. The flow chart of case/control group including and excluding. 
Table I. Baseline characteristics.

\begin{tabular}{lll}
\hline & \multicolumn{2}{l}{ All subjects, N(\%) } \\
\hline Number & 375243 & \\
Age, mean (years old) & 43.93 & \pm 16.67 \\
Urbanicity (\%) & 292646 & $(77.99 \%)$ \\
Health related behaviors & & \\
Areca nut chewing rate (\%) & 14.74 & $\pm 6.72 \%$ \\
Smoking rate (\%) & 43.69 & $\pm 4.84 \%$ \\
Infrequent vegetable eating (\%) & 2.07 & $\pm 0.59 \%$ \\
Exercise rate (\%) & 60.22 & $\pm 5.66 \%$ \\
Medical history & & \\
Diabetes mellitus (\%) & 25313 & $(6.75 \%)$ \\
Hypertension (\%) & 55550 & $(14.80 \%)$ \\
Congestive heart failure (\%) & 3990 & $(1.06 \%)$ \\
Ischemic stroke (\%) & 6546 & $(1.74 \%)$ \\
Ischemic heart disease (\%) & 18974 & $(5.06 \%)$ \\
Chronic obstructive pulmonary & 31119 & $(8.29 \%)$ \\
disease (\%) & & \\
Thyroid disease (\%) & 1551 & $(0.41 \%)$ \\
Valvular heart disease (\%) & 4203 & $(1.12 \%)$ \\
Pulmonary heart disease (\%) & 205 & $(0.05 \%)$ \\
Sleep apnea (\%) & 14134 & $(3.77 \%)$ \\
Renal failure, chronic (\%) & 3515 & $(0.94 \%)$ \\
Cardiomyopathy (\%) & 350 & $(0.09 \%)$ \\
Atrial fibrillation (\%) & 1326 & $(0.35 \%)$ \\
CHADS2- scorea & & \\
0 & 297499 & $(79.28 \%)$ \\
1 & 49063 & $(13.07 \%)$ \\
2 & 19316 & $(5.15 \%)$ \\
3 & 6052 & $(1.61 \%)$ \\
4 & 2594 & $(0.69 \%)$ \\
5 & 669 & $(0.18 \%)$ \\
6 & 50 & $(0.01 \%)$ \\
\hline & & \\
\hline
\end{tabular}

${ }^{a}$ CHADS2- score, please see reference [20].

\section{Validation of the AF diagnosis}

To validate the diagnostic accuracy, a review of medical charts was performed by a physician who was blinded in this study. One hundred charts which showed the ICD9 code 427.31 or 427.32 and the use of medications such as amiodarone, propafenone, digoxin, aspirin, clopidogrel, or warfarin (any of which might be prescribed to AF patients in out-patient departments or wards) were randomly selected in one medical centre and one community teaching hospital to see if there was any record of AF either by electrocardiogram or 24-hour Holter monitoring. The diagnostic accuracy of AF (by ICD9 coding) was found to be $98 \%(100 \%(50 / 50)$ and $96 \%(48 / 50)$ in the medical center and community teaching hospital, respectively. Patients who had an AF diagnosis by ICD9 code and also used the aforementioned medications in the same index date were selected for inclusion in the case group. Those patients who had an AF diagnosis by ICD9 code but had never been medicated (suspected
AF) were excluded from the study (Figure 1).

\section{National Health Interview Survey}

The 2005 NHIS in Taiwan was conducted by the National Health Research Institutes, National Bureau of Controlled Drugs and Bureau of Health Promotion, Department of Health, Taiwan[19]. The study sample was nationally representative by using a multi-stage stratified systematic sampling design. All subjects were personally interviewed by well-trained interviewers. The detailed data was then released for public use and can be collected from web site: Year 2005 National Health Interview Survey http://olap.bhp.doh.gov.tw/en_US/Search/Search.a spx?menu=100000000006\&KeyWord=betel. The target population of the original survey was $22,615,307$ individuals whose households were registered in one of the 23 counties or cities in Taiwan in the year 2004-2005. Among the 187 neighbourhoods or villages, 30,680 persons were sampled (sampling rate $1.35 \%$ ). A total of 24,726 persons completed the survey with a response rate of $80.6 \%$. Individuals younger than 18 years of age $(n=6198)$, females older than 18 years of age ( $\mathrm{n}=9101)$, and those living outside of the main island of Taiwan $(n=302)$ were excluded from this study. The health related behaviors of the total 9,125 participants were used as exposure variables in our study. The particular health related behaviors included in our analysis were cigarette smoking, betel nut chewing, infrequent vegetable eating, and exercising. The habitual behaviors were identified by questionnaire; "Have you ever smoked cigarettes?"; "Do you chew betel quid now?" ; "During the past 2 weeks, have you ever performed any exercise?" and "Do you eat fresh vegetable less than or equal to once in a week?". The information of health related behaviors were presented as a percentage of the population of one county or city.

\section{Statistical analysis}

Data were expressed as mean \pm standard deviation or percentage. The average rate of health related behaviors was computed by the sum of subjects in cities/counties multiplied by the rate of corresponding health related behavior in that city/county divided by the numbers of all subjects. The data of health related behavior were collected from the NHIS by percentage of city/county units, the remaining information of the other confounding factors (such as diabetes, hypertension) were collected from the NHIRD personal data.

The correlation between AN using rate and prevalence of AF in a city/county of Taiwan were analyzed by continuous variable or quartiles of different rate/ratio. Spearman`s coefficient and $R$ 
squared were obtained as indicators of the degree of linearity between the two variables. The spatial analysis software OpenGeoDa 1.0.1 was used for demonstration of the geographic relationship between AN using rate and prevalence of AF in cities/counties of Taiwan.

Furthermore, we used unconditional logistic regression to analyze the effect of health related behaviors on AF risk. Demographic characteristics, health related behaviors, and prevalence of disease were compared between Non-AF and AF patients. The covariates included age, urbanization, rate of cigarette smoking, rate of infrequent vegetable eating, rate of exercising, presence of diabetes mellitus, hypertension, congestive heart failure, ischemic stroke, chronic obstructive pulmonary disease (COPD), thyroid disease, valvular heart disease, pulmonary heart disease, sleep apnea, chronic renal failure, and cardiomyopathy.

Three multivariate models were used to explore the relationship between AN chewing and the risk of AF. Model 1was only adjusted for the demographic characteristics and rate of health related behaviors. Model 2 was adjusted for the demographic characteristics, rate of health related behaviors, and the covariates which were not reported to be associated with AN chewing previously. Model 3 was adjusted for all the covariates. All tests were 2-sided and the level of significance was established as $\mathrm{P}<0.05$. All statistical analyses were carried out by using SAS software (version 8.0; SAS Institute Inc, Cary, NC).

\section{Results}

In total, 1,000,000 subjects were in the 2005 NHIRD. After females, males $<18$ years of age, and subjects who lived in off-shore island were excluded, there were 375,243 males in the final analysis (Figure 1). Of the 375,243 males (mean age, 44 years old), 1326 $(0.35 \%)$ had the diagnosis of AF. The average rates of health related behaviors including AN chewing, cigarette smoking, infrequent vegetable eating, and exercising according to 2005 NHIS were $14.74 \%, 43.69 \%$, $2.07 \%$, and $60.22 \%$ respectively (Table 1 ). The prevalence of other medical diseases ranged from $0.05 \%$ for pulmonary heart disease to $14.8 \%$ for hypertension. For CHADS2 score[20], the majority of study subjects had scores $0-2$.

The relationship between rate of AN chewing in a city/county and the prevalence of AF was evaluated in Figure 2. The scatter plot of these two showed a linear relationship. The Spearman`s correlation coefficient between the rate of AN chewing and the prevalence of AF was 0.558 , which was significant $(\mathrm{p}=$
0.007 ) (correlation coefficient $=0.733, \mathrm{p}<0.001, \mathrm{R}^{2}=$ 0.538 in Pearson method).

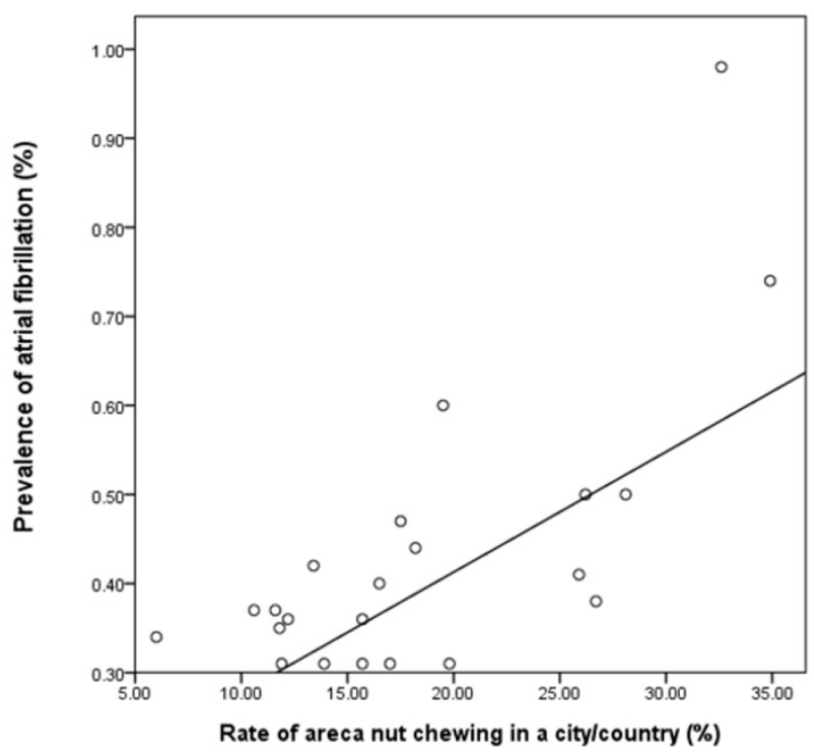

Figure 2. Scatter plot of city/county according to rate of AN chewing and the prevalence of AF. AF, atrial fibrillation; AN, areca nut. The scatter plot of city/country according to rate of AN chewing and the prevalence of AF showed a linear relationship (correlation coefficient $=0.733, p<0.00 I, R^{2}=$ 0.538 in Pearson method) (correlation coefficient $=0.558, p=0.007, R^{2}=$ 0.538 in Spearman`s method).

The two Taiwan maps were created according to the quantile of $\mathrm{AN}$ chewing rate and prevalence of $\mathrm{AF}$ in our dataset respectively. Figure 3 showed the high degree of overlap between the rate of AN chewing and prevalence of $\mathrm{AF}$ in cities/counties by quartile.

After adjusting for other covariates, we found that the AN chewing rate was independently positively associated with the prevalence of AF (Table 2). The adjusted odd ratio (OR) of current AN chewing was $1.02(95 \%$ confidence interval $(\mathrm{CI})=1.00-1.04)$ in the risk of AF prevalence in Model 3. Similar results were found in Model 1 and 2. Other covariates, including age, hypertension, congestive heart failure, ischemic stroke, ischemic heart disease, COPD, thyroid disease, valvular heart disease, and cardiomyopathy, were all significantly associated with $\mathrm{AF}$ in Model 3 (Table 2).

We also analysed the case group as AF diagnosed by ICD-9 coding only but not confirmed by medication use as previously mentioned (ICD-9 AF) with the control group (No ICD9-AF) and the same result of significantly association between $\mathrm{AN}$ and $\mathrm{AF}$ was found $(\mathrm{OR}=1.02,95 \% \mathrm{CI}=1.00-1.04, \mathrm{p}=0.016)$. Furthermore, urbanization was found to be positively associated with the prevalence of AF. The adjusted OR for urbanization in full adjusted model was 1.29 (95\% CI, 1.10-1.51) in risk of AF prevalence. 
Table 2. Odds ratio for atrial fibrillation associated with areca nut chewing and other risk factors (model I, 2, 3).

\begin{tabular}{|c|c|c|c|c|c|c|c|}
\hline & & & & Male & & & \\
\hline & $\begin{array}{l}\mathrm{AF} \\
(\mathrm{n}=1326)\end{array}$ & $\begin{array}{l}\text { Non-AF } \\
(\mathrm{n}=373917)\end{array}$ & Crude OR & $\begin{array}{l}\text { Model 1a } \\
\text { Adjusted OR }\end{array}$ & $\begin{array}{l}\text { Model 2b } \\
\text { Adjusted OR }\end{array}$ & $\begin{array}{l}\text { Model 3c } \\
\text { Adjusted OR }\end{array}$ & P value \\
\hline \multicolumn{8}{|l|}{ Health related behaviors } \\
\hline Areca nut chewing rate $(\%)$ & $15.84 \pm 7.39 \%$ & $14.74 \pm 6.72 \%$ & $1.02(1.02-1.03)$ & $1.02(1.00-1.03)$ & $1.02(1.00-1.04)$ & $1.02(1.00-1.04)$ & 0.016 \\
\hline Smoking rate $(\%)$ & $44.23 \pm 4.89 \%$ & $43.68 \pm 4.84 \%$ & $1.02(1.01-1.04)$ & $1.00(0.98-1.02)$ & $1.00(0.98-1.02)$ & $1.00(0.98-1.02)$ & 0.94 \\
\hline $\begin{array}{l}\text { Infrequent vegetable eating } \\
(\%)\end{array}$ & $2.08 \pm 0.65 \%$ & $2.07 \pm 0.59 \%$ & $1.03(0.94-1.13)$ & $0.97(0.89-1.06)$ & $0.98(0.90-1.08)$ & $0.99(0.90-1.08)$ & 0.77 \\
\hline Exercise (\%) & $59.54 \pm 5.94 \%$ & $60.22 \pm 5.66 \%$ & $0.98(0.97-0.99)$ & $1.01(0.99-1.02)$ & $1.01(0.99-1.02)$ & $1.00(0.99-1.02)$ & 0.66 \\
\hline Age, mean (year old) & $69.86 \pm 12.68$ & $43.84 \pm 16.62$ & $1.09(1.08-1.09)$ & $1.09(1.08-1.09)$ & $1.07(1.07-1.08)$ & $1.06(1.05-1.06)$ & $<0.001$ \\
\hline Urbanicity (\%) & $965(72.8 \%)$ & $291681(78 \%)$ & $0.75(0.67-0.85)$ & $1.25(1.07-1.45)$ & $1.30(1.11-1.52)$ & $1.29(1.10-1.51)$ & 0.001 \\
\hline Diabetes mellitus (\%) & $269(20.3 \%)$ & $25044(6.7 \%)$ & $3.55(3.10-4.06)$ & & & $0.96(0.83-1.11)$ & 0.61 \\
\hline Hypertension (\%) & $816(61.5 \%)$ & $54734(14.6 \%)$ & $9.33(8.35-10.43)$ & & & $1.72(1.51-1.95)$ & $<0.001$ \\
\hline Congestive heart failure (\%) & $421(31.8 \%)$ & $3569(1.0 \%)$ & $\begin{array}{l}48.27 \\
(42.80-54.44)\end{array}$ & & $7.75(6.73-8.93)$ & $6.03(5.23-6.95)$ & $<0.001$ \\
\hline Ischemic stroke (\%) & $222(16.7 \%)$ & $6324(1.7 \%)$ & $\begin{array}{l}11.69 \\
(10.09-13.53)\end{array}$ & & & $2.23(1.90-2.62)$ & $<0.001$ \\
\hline Ischemic heart disease (\%) & $599(45.2 \%)$ & $18375(4.9 \%)$ & $\begin{array}{l}15.95 \\
(14.30-17.79)\end{array}$ & & & $2.47(2.17-2.81)$ & $<0.001$ \\
\hline $\begin{array}{l}\text { Chronic obstructive pulmo- } \\
\text { nary disease }(\%)\end{array}$ & $482(36.4 \%)$ & $30637(8.2 \%)$ & $6.40(5.72-7.16)$ & & $1.53(1.34-1.74)$ & $1.36(1.19-1.54)$ & $<0.001$ \\
\hline Thyroid disease (\%) & $40(3.0 \%)$ & $1511(0.4 \%)$ & 7.67 (5.57-10.54) & & $\begin{array}{l}7.68 \\
(5.40-10.93)\end{array}$ & $\begin{array}{l}7.17 \\
(5.02-10.24)\end{array}$ & $<0.001$ \\
\hline Valvular heart disease (\%) & $242(18.3 \%)$ & $3961(1.1 \%)$ & $\begin{array}{l}20.85 \\
(18.08-24.05)\end{array}$ & & $5.12(4.38-6.08)$ & $4.29(3.64-5.05)$ & $<0.001$ \\
\hline Pulmonary heart disease (\%) & $11(0.8 \%)$ & $194(0.1 \%)$ & $16.12(8.76-29.66)$ & & $1.21(0.62-2.39)$ & $1.31(0.67-2.57)$ & 0.43 \\
\hline Sleep apnea & $140(10.6 \%)$ & 13994(3.7\%) & $3.04(2.55-3.62)$ & & $1.05(0.87-1.27)$ & $0.90(0.74-1.09)$ & 0.26 \\
\hline Renal failure, chronic & $88(6.6 \%)$ & $3427(0.9 \%)$ & $7.69(6.18-9.57)$ & & $1.26(0.99-1.60)$ & $1.06(0.84-1.35)$ & 0.61 \\
\hline Cardiomyopathy (\%) & $47(3.5 \%)$ & $303(0.1 \%)$ & $\begin{array}{l}45.32 \\
(33.17-61.92)\end{array}$ & & $5.55(3.81-8.09)$ & $5.13(3.54-7.43)$ & $<0.001$ \\
\hline
\end{tabular}

AF, atrial fibrillation; OR, odd ratio; The different covariates were analyzed in different models from Model 1to 3. a age, urbanicity, health behavior (areca nut chewing, smoking, exercise, and infrequent vegetable eating); $b$ all covariates in model 1 and heart failure, chronic obstructive pulmonary disease, thyroid disease, vulvular heart disease, pulmonary heart disease, sleep apnea, renal failure, and cardiomyopathy; ${ }^{c}$ all covariates in model 2 and diabetes, hypertension, ischemic heart disease, and ischemic stroke. ${ }^{\mathrm{d}} \mathrm{p}$ valve for model 3.

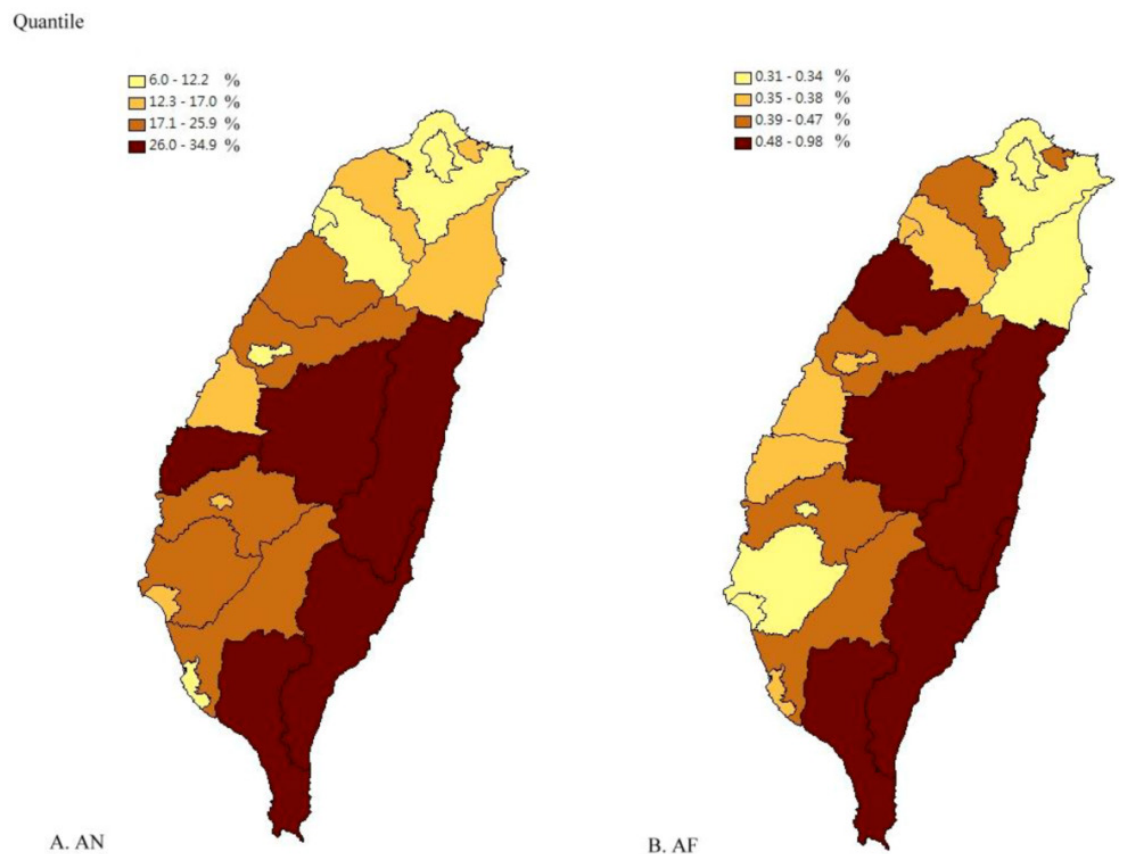

Figure 3. The comparison of $A N$ chewing rate and $A F$ prevalence in Taiwan. $A F$, atrial fibrillation; $A N$, areca nut. (A) $A N$ chewing rate in city/county in Taiwan. (B) AF prevalence in city/county in Taiwan. The quantile of AN is according to the equal proportions of AN chewing rate in 22 different cities/counties in Taiwan. The quantile of AF prevalence is according to the proportions of AF prevalence in 22 different cities/counties in Taiwan. 


\section{Discussion}

The present study mainly found that AN chewing was associated with the increased prevalence of $\mathrm{AF}$ in Taiwanese men. Other health related behaviors such as smoking, exercise, and infrequent vegetable eating were not associated with an increased prevalence of AF in our study. To our best knowledge, this is the first study to demonstrate the association between rate of $\mathrm{AN}$ chewing and prevalence of $\mathrm{AF}$.

According to the 2005 NHIS dataset and the 2005 NHIRD, we found a trend that the higher the rate of current $\mathrm{AN}$ chewing, the higher the prevalence of $\mathrm{AF}$ in a city/county. The scatter plot and linear regression of the city/county according to rate of AN chewing and the prevalence of AF showed the linear relationship between these. The two maps of cities/counties of Taiwan showed similar distributions between AN chewing rate and prevalence of AF in quantile. The prevalence of $\mathrm{AF}$ in our study was $0.35 \%$. The lower prevalence rate of $\mathrm{AF}$ maybe due to the relative young male population in our study (mean 43.9 year old) and this was compatible with previous studies [1, 3]. After adjusting the conventional and novel risk factors of $\mathrm{AF}$, the rate of current $\mathrm{AN}$ chewing was still significantly associated with prevalence of AF. The rates of smoking, infrequent vegetable eating, and exercising were not associated with prevalence of AF. Urbanization was positively associated with AF, which may due to differences in medical care resource and socio-economic status between urban and rural areas in Taiwan.

Several previous studies have reported the association between AN chewing and the risk of cardiac arrhythmias [11-16, 21], but most of these previous studies were case reports or case series. Wyatt first reported that betel nut chewing in 28 young male operators resulted in significant increase in pulse rate. The most common cardiac arrhythmias related to betel chewing were sinus tachycardia, and premature atrial contractions [21]. Chiang et al. then reported one sudden death and one paroxysmal supraventricular tachycardia case resulting from chewing betel quid [16]. Subsequently, Deng et al. reviewed the probable or possible betel nut related toxicity cases in 1988-1998 reported to the Taiwan Poison Control Center. The most common manifestations were tachycardia/palpitations, tacypnea/dyspnea, hypotension, sweating, dizziness, chest discomfort, coma, acute myocardial infarction, and ventricular fibrillation. Betel nut chewing can produce significant cholinergic, neurological, cardiovascular, and gastrointestinal manifestations [14]. In volume 85 of International Agency for Research on Cancer (IARC) report, extract of Piper Betle inflorescence, a composition of common AN chewing regimen induced hypotensive and bradycardiac effects in male rats by intra-arterial and intrathecal injections. The acute administration of extracts of betel inflorescence by different routes might activate C-fiber-evoked parasympathetic and sympathetic cardiovascular reflexes in rats [13]. Dose-dependent atropine-sensitive inhibition of isolated guinea-pig atrial contractility was found by Ghayur et al [12]. Most recently, Chou et al. reported that Pinang-Wang, a special form of betel nut, induced persisting ST elevation in electrocardiogram with normal coronary artery by angiography and refractory ventricular tachycardia/fibrillation in a 50 years old male. The ventricular arrhythmias were refractory to conventional treating algorithm but only converted by the intravenous injection of sodium bicarbonate. Thus, the constituents of Pinang-Wang might possess the property of sodium channel blocker [11].

$\mathrm{AF}$, one of the most common cardiac arrhythmias in the world, is thought to be associated with ageing, hypertension, symptomatic heart failure, tachycardiomyopathy, valvular heart disease, cardiomyopathies, atrial septal defect, coronary artery disease, thyroid dysfunction, obesity, COPD, sleep apnea, and chronic kidney disease [22]. AN chewing has been previously reported to be associated with some risk factors of AF. General and central obesity were related to AN chewing in Chinese males reported by Lin et al [8]. AN chewing has also been associated with HTN in Taiwanese patients with type-2 diabetes [5]. In our previous study, AN chewing was independently associated with CAD [10]. Those findings, along with ours, suggest that AN might act as an independent risk factor for $\mathrm{AF}$ or as a mediator that modifies the risk of hypertension, obesity, or CAD.

There are several possible mechanisms to explain the link between AN chewing and AF. AF was thought to be related to chronic inflammation and increased sympathetic/parasympathetic activity in previous studies [23]. AN and the other component of regimens of betel nut chewing were associated with inflammation by the mechanisms as increasing COX-2, reactive oxygen species, superoxide dismutase, NF-KB, tumor necrosis factor-alpha, interleukin (IL)-1 beta, IL-6 and IL-8. Arecoline, the most well-known content of the AN, was reported to induce COX-2 up-regulation [24]. Hydroxychavicol, another major phenolic compound in the inflorescence, could induce reactive oxygen species production via redox cycling [25], increase superoxide dismutase activity in mice liver [26]. Ni et al. found 
that human buccal mucosa cells exposed to AN could activate NF- $\mathrm{kB}$ expression [27]. The expressions of tumor necrosis factor-alpha, interleukin (IL)-1 beta, IL-6 and IL-8 were also increased in human peripheral blood mononuclear cells after being treated with AN extract [28]. AN chewing was previously reported to increase sympathetic and parasympathetic activity $[13,29]$. Ghayur et al.had reported the cardio-selective cholinomimetic activity of the betel nut crude extract in isolated guinea-pig atrium [12]. The cholinomimetic and acetylcholinesterase (AChE) inhibitory constituents in betel nut used in rabbit were reported by Gilani et al[30]. The increasing consumption of betel nut preparation resulted in a reduction in RR interval variation in electrocardiogram, particularly during deep breathing in human subjects [31].

This study had several limitations. First, this is an ecological study. The exposure of interest, AN chewing, and several other covariates such cigarette smoking, infrequent vegetable eating, and exercise were not individual information hence the introduction of confounding bias is likely. Second, since the design was cross-sectional, we cannot infer the causality between AN and AF. Third, the diagnosis of AF and other co-morbidities may not always be accurate. However, we have attempted to overcome this by verifying the diagnoses in one medical center and one community teaching hospital.

\section{Conclusions}

In conclusion, we have shown that rate of current $\mathrm{AN}$ chewing is independently associated with the prevalence of AF in Taiwanese men. The rate of smoking, infrequent vegetable eating, and level of exercise were not associated with the prevalence of AF. Further studies are needed to clarify the dose responsive relationship between AN chewing and AF and the mechanisms behind that correlation.

\section{Supplementary Material}

Supplementary Table 1

http://www.medsci.org/v10p0804s1.pdf

\section{Acknowledgements}

We are grateful for the data from the National Health Interview Survey Original Database provided by the Bureau of Health Promotion and Taiwan Bureau of National Health Insurance, Department of Health, National Health Research Institutes. The interpretation and conclusions in the present study do not represent those of Bureau of Health Promotion and Taiwan Bureau of National Health Insurance, Department of Health, National Health Research Institutes. The authors thank the help from the Statisti- cal Analysis Laboratory, Department of Internal Medicine, Kaohsiung Medical University Hospital.

\section{Competing Interests}

The authors have declared that no competing interest exists.

\section{References}

1. Go AS, Hylek EM, Phillips KA, Chang Y, Henault LE, Selby JV, et al. Prevalence of Diagnosed Atrial Fibrillation in Adults. JAMA: The Journal of the American Medical Association. 2001; 285: 2370-5. doi:10.1001/jama.285.18.2370.

2. Benjamin EJ, Wolf PA, D'Agostino RB, Silbershatz H, Kannel WB, Levy D. Impact of Atrial Fibrillation on the Risk of Death : The Framingham Heart Study. Circulation. 1998; 98: 946-52. doi:10.1161/01.cir.98.10.946.

3. Chien K-L, Su T-C, Hsu H-C, Chang W-T, Chen P-C, Chen M-F, et al. Atrial fibrillation prevalence, incidence and risk of stroke and all-cause death among Chinese. International Journal of Cardiology. 2010; 139: 173-80. doi:10.1016/j.ijcard.2008.10.045.

4. Lee CH, Ko AMS, Warnakulasuriya S, Yin BL, Sunarjo, Zain RB, et al. Intercountry prevalences and practices of betel-quid use in south, southeast and eastern asia regions and associated oral preneoplastic disorders: An international collaborative study by asian betel-quid consortium of south and east Asia. International Journal of Cancer. 2011; 129: 1741-51. doi:10.1002/ijc.25809.

5. Tseng $\mathrm{CH}$. Betel nut chewing is associated with hypertension in Taiwanese type 2 diabetic patients. Hypertens Res. 2008; 31: 417-23. doi:JST.JSTAGE/hypres/31.417 [pii].

6. Lan TY, Chang WC, Tsai YJ, Chuang YL, Lin HS, Tai TY. Areca nut chewing and mortality in an elderly cohort study. Am J Epidemiol. 2007; 165: 677-83. doi:kwk056 [pii]. 10.1093/aje/kwk056.

7. Guh JY, Chuang LY, Chen HC. Betel-quid use is associated with the risk of the metabolic syndrome in adults. Am J Clin Nutr. 2006; 83: 1313-20. doi:83/6/1313 [pii].

8. Lin WY, Pi-Sunyer FX, Liu CS, Li TC, Li CI, Huang CY, et al. Betel nut chewing is strongly associated with general and central obesity in Chinese male middle-aged adults. Obesity (Silver Spring). 2009; 17: 1247-54. doi:oby200938 [pii]. 10.1038/oby.2009.38.

9. Yen AM, Chen LS, Chiu YH, Boucher BJ, Chen TH. A prospective community-population-registry based cohort study of the association between betel-quid chewing and cardiovascular disease in men in Taiwan (KCIS no. 19). Am J Clin Nutr. 2008; 87: 70-8. doi:87/1/70 [pii].

10. Tsai WC, Wu MT, Wang GJ, Lee KT, Lee CH, Lu YH, et al. Chewing areca nut increases the risk of coronary artery disease in Taiwanese men: a case-control study. BMC Public Health. 2012; 12: 162. doi:10.1186/1471-2458-12-162.

11. Chou C-J, Su H-M, Lee H-H, Ko Y-C, Chen P-H, Chen B-H. Life-Threatening Cardiac Toxicity After Chewing Inverted Nut (Pinang-Wang). Annals of emergency medicine. 2009; 54: 757-8.

12. Ghayur MN, Gilani AH. Cardio-selective inhibitory effect of the betel nut extract: possible explanation. Pharmazie. 2007; 62: 67-71.

13. IARC Working Group on the Evaluation of Carcinogenic Risks to Humans. Betel-quid and areca-nut chewing and some areca-nut derived nitrosamines. IARC Monogr Eval Carcinog Risks Hum. 2004; 85: 1-334.

14. Deng JF, Ger J, Tsai WJ, Kao WF, Yang CC. Acute toxicities of betel nut: rare but probably overlooked events. Journal of toxicology Clinical toxicology. 2001; 39: 355-60.

15. Nelson BS, Heischober B. Betel nut: a common drug used by naturalized citizens from India, Far East Asia, and the South Pacific Islands. Ann Emerg Med. 1999; 34: 238-43.

16. Chiang WT, Yang CC, Deng JF, Bullard M. Cardiac arrhythmia and betel nut chewing--is there a causal effect? Vet Hum Toxicol. 1998; 40: 287-9.

17. [Internet] National Health Insurance Research Database. http://w3.nhri.org.tw/nhird//en/index.htm.

18. NHIS working group. 2005 Taiwan National Health Interview and Medication Survey, Characteristics of completed sample (In Chinese). Taiwan National Health Interview Survey Research Brief, Taipei. 2006.

19. Ko YC, Chiang TA, Chang SJ, Hsieh SF. Prevalence of betel quid chewing habit in Taiwan and related sociodemographic factors. Journal of oral pathology \& medicine : official publication of the International Association of Oral Pathologists and the American Academy of Oral Pathology. 1992; 21: 261-4. 
20. Lin L-Y, Lee C-H, Yu C-C, Tsai C-T, Lai L-P, Hwang J-J, et al. Risk factors and incidence of ischemic stroke in Taiwanese with nonvalvular atrial fibrillation--A nation wide database analysis. Atherosclerosis. 2011; 217: 292-5.

21. Wyatt TA. Betel nut chewing and selected psychophysiological variables. Psychological reports. 1996; 79: 451-63.

22. Association DwtscotEHR, Surgery EbtEAfC-T, Members ATF, Camm AJ, Kirchhof P, Lip GYH, et al. Guidelines for the management of atrial fibrillation. European Heart Journal. 2010; 31: 2369-429. doi:10.1093/eurheartj/ehq278.

23. Fuster V, Rydén LE, Cannom DS, Crijns HJ, Curtis AB, Ellenbogen KA, et al. ACC/AHA/ESC 2006 Guidelines for the Management of Patients With Atrial Fibrillation-Executive Summary. Circulation. 2006; 114: 700-52. doi:10.1161/circulationaha.106.177031.

24. Tsai C-H, Chou M-Y, Chang Y-C. The up-regulation of cyclooxygenase-2 expression in human buccal mucosal fibroblasts by arecoline: a possible role in the pathogenesis of oral submucous fibrosis. Journal of Oral Pathology \& Medicine. 2003; 32: 146-53.

25. Iverson SL, Hu LQ, Vukomanovic V, Bolton JL. The influence of the p-alkyl substituent on the isomerization of o-quinones to p-quinone methides: potential bioactivation mechanism for catechols. Chem Res Toxicol. 1995; 8: 537-44.

26. Choudhary D, Kale RK. Antioxidant and non-toxic properties of Piper betle leaf extract: in vitro and in vivo studies. Phytotherapy Research. 2002; 16: 461-6.

27. Ni W-F, Tsai C-H, Yang S-F, Chang Y-C. Elevated expression of NF-kB in oral submucous fibrosis - Evidence for NF-KB induction by safrole in human buccal mucosal fibroblasts. Oral oncology. 2007; 43: 557-62.

28. Chang LY, Wan HC, Lai YL, Kuo YF, Liu TY, Chen YT, et al. Areca nut extracts increased expression of inflammatory cytokines, tumor necrosis factor- $\alpha$, interleukin- $1 \beta$, interleukin- 6 and interleukin- 8 , in peripheral blood mononuclear cells. Journal of Periodontal Research. 2009; 44: 175-83. doi:10.1111/j.1600-0765.2008.01104.x.

29. Chu NS. Effects of Betel chewing on the central and autonomic nervous systems. Journal of biomedical science. 2001; 8: 229-36.

30. Gilani AH, Ghayur MN, Saify ZS, Ahmed SP, Choudhary MI, Khalid A. Presence of cholinomimetic and acetylcholinesterase inhibitory constituents in betel nut. Life Sciences. 2004; 75: 2377-89. doi:10.1016/j.lfs.2004.03.035.

31. Chu NS. Effect of betel chewing on RR interval variation. Journal of the Formosan Medical Association = Taiwan yi zhi. 1995; 94: 106-10. 